\title{
Antimicrobial Benzyltetrahydroisoquinoline-Derived Alkaloids from the Leaves of Doryphora aromatica
}

\author{
Miaomiao Liu,* Jianying Han, Yunjiang Feng, Gordon Guymer, Paul I. Forster, and Ronald J. Quinn*
}

Cite This: J. Nat. Prod. 2021, 84, 676-682

Read Online

ABSTRACT: Four new alkaloids, $(R)$-nomimantharine trifluoroacetate (2), 12-demethylphaeantharine trifluoroacetate (3), nominanthranal trifluoroacetate (4), and the enolic form of 1hydroxy-6,7-dimethoxy-2-methylisoquinoline trifluoroacetate (5), together with the known dimeric alkaloid phaeantharine trifluoroacetate (1), have been isolated from the extract of the leaves of the rainforest tree Doryphora aromatica (Monimiaceae). The structures of these compounds were elucidated by HRMS and

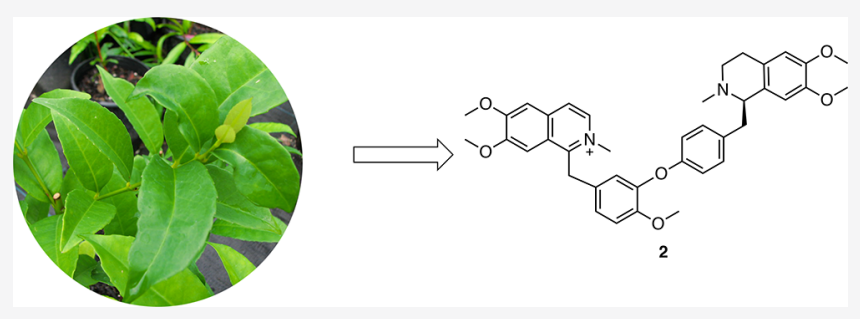
1D and 2D NMR data. (R)-Nomimantharine trifluoroacetate (2) contains an ether linkage connecting a benzylisoquinoline unit with a tetrahydroisoquinoline, a novel class of dimeric alkaloid. The absolute configuration of $(R)$-nomimantharine trifluoroacetate (2) was established via electronic circular dichroism data. The compounds isolated were subjected to in vitro antimicrobial assays against a panel of pathogenic microorganisms, including Mycobacterium smegmatis, M. tuberculosis, Escherichia coli, Staphylococcus aureus (SA), and five clinical isolates of oxacillin/methicillinresistant S. aureus (MRSA). Phaeantharine trifluoroacetate (1) and (R)-nomimantharine trifluoroacetate (2) showed moderate inhibitory activities against Mycobacteria and MRSA strains.

Since the discovery of penicillin, antibiotics have saved $\checkmark$ millions of lives every year. However, the advent of drug resistance has rendered many of them ineffective against organisms such as methicillin-resistant Staphylococcus aureus (MRSA) and multidrug- and extensively drug-resistant Mycobacterium tuberculosis. ${ }^{1,2} \mathrm{New}$ drugs that retain potency against multidrug/extensively drug-resistant strains with the additional benefit of a shortened treatment duration and ease of administration are urgently needed. Natural products (NPs) are superior starting points for the major antimicrobials used in the clinic. ${ }^{3,4}$ Antimicrobial NPs can be obtained from microorganisms and plants. ${ }^{5-9}$

The traditional process of discovering new bioactive natural products via bioassay-guided isolation is generally long and laborious, and known natural products are frequently rediscovered. This work reports a combination of HPLC fractionation and an NMR fingerprint technique to discover compounds with new structures. We have previously reported a strategy to generate a natural product fraction library with lead- and drug-like constituents by selecting favorable physicochemical properties such as $\log \mathrm{P}<5$. $^{10,11}$ The whole library (202 983 fractions) was evaluated by a phenotypic highthroughput screening (HTS) assay against M. tuberculosis $\mathrm{H} 37 \mathrm{Rv}$ and resulted in a series of active fractions. As part of a strategy to build an NMR database of fractions, 748 biota, including 291 marine organisms and 457 plant samples, were re-extracted and refractionated with collection of five fractions per extract to give 3740 fractions according to the reported protocol. ${ }^{10}$ Each fraction in the library was evaluated by comparison of NMR spectra to achieve NMR fingerprinting to aid the identification of new constituents (Figure 1). The NMR spectrum of a fraction is a fingerprint of its entire chemical composition and, therefore, never lies about the

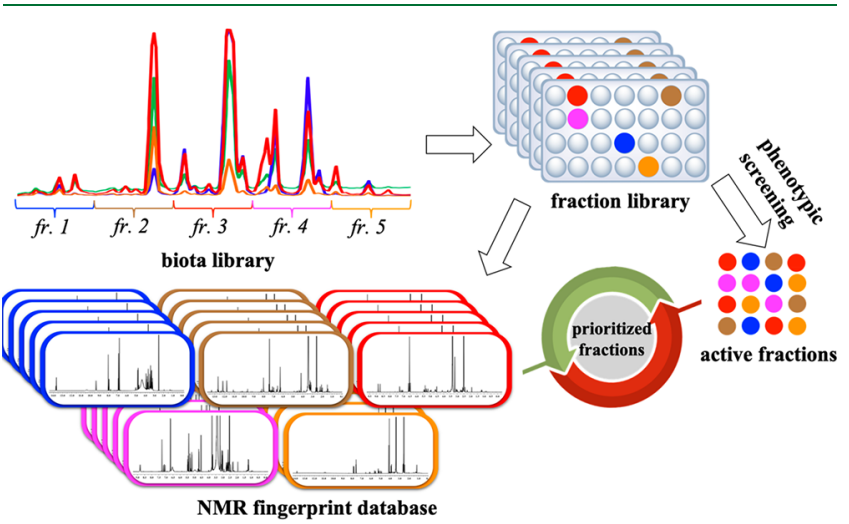

Figure 1. Overview of natural product drug discovery using both phenotypic screening and NMR fingerprinting.

Special Issue: Special Issue in Honor of A. Douglas Kinghorn

Received: October 12, 2020

Published: March 5, 2021

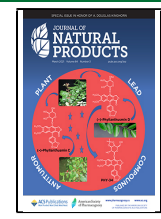



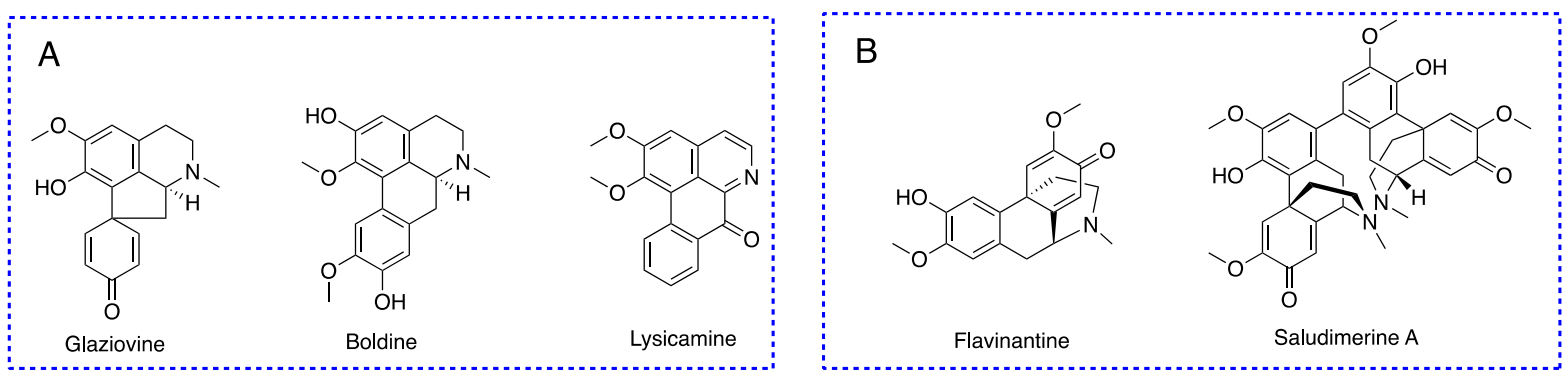

Figure 2. (A) Structures of proaporphine, aporphine, and oxoaporphine alkaloids. (B) Structures of flavinantine and saludimerines.

composition of fractions. ${ }^{12}$ The aim of NMR fingerprintingguided NP discovery is to detect as many compounds as possible, which means maintaining high detection sensitivity. The advent of high-field instruments together with cryoprobes and small volume tubes ( 3 or $1.7 \mathrm{~mm}$ NMR tubes) has addressed the previous limitation of low sensitivity so that NMR spectra of fractions can be directly analyzed to identify constituents. Since most secondary metabolites contain hydrogen, the ${ }^{1} \mathrm{H}$ NMR spectrum of samples containing a large number of metabolites would result in extensive overlap in signals in the NMR fingerprint. The NMR fingerprint of fractions gave spectra with sufficient resolution to allow compound isolation to be monitored with the objective to isolate all constituents in the fraction by ensuring all signals in the fraction were present within one or more of the isolated compounds. With the guidance of NMR fingerprinting, we proceeded to isolate active compounds in one of the prioritized active fractions (Figure 1).

In this study, we report compound isolation and identification from an active fraction prioritized from the phenotypic HTS screening in combination with NMR fingerprinting. The fraction was derived from a sample of the rainforest tree Doryphora aromatica (F.M.Bailey) L.S.Sm. (family Monimiaceae), which belongs to the order Laurales sensu Dahlgren and comprises about 28 genera and 195-200 species. ${ }^{13}$ Monimiaceae is a family rich in benzyltetrahydroisoquinoline-derived proaporphine, aporphine, and oxoaporphine alkaloids (Figure 2A). ${ }^{14,15}$ Less common is the presence of morphinanedienone-type compounds, such as flavinantine and saludimerines (Figure 2B). ${ }^{16,17}$ In our current study, five benzyltetrahydroisoquinoline-derived alkaloids, including four new members, were isolated from Doryphora aromatica. While bisbenzylisoquinolines (1) and bistetrahydroisoquinolines are known, $(R)$-nomimantharine trifluoroacetate (2) contains an ether linkage connecting a benzylisoquinoline unit and a tetrahydroisoquinoline unit, a novel class of dimeric alkaloid. Compounds were assayed against a panel of pathogenic microorganisms, and phaeantharine trifluoroacetate (1) and (R)-nomimantharine trifluoroacetate (2) exhibited activities against Mycobacteria and MRSA strains.

\section{RESULTS AND DISCUSSION}

The leaves of $D$. aromatica were extracted using a 95\% EtOH solution followed by Sephadex LH-20 fractionation. Analysis of the NMR fingerprints of all fractions identified an enriched alkaloid fraction. The latter was fractionated by semipreparative HPLC, resulting in the isolation of major compounds $\mathbf{1}$ and $\mathbf{2}$ as well as the minor secondary metabolites 3-5 (Figure 3).

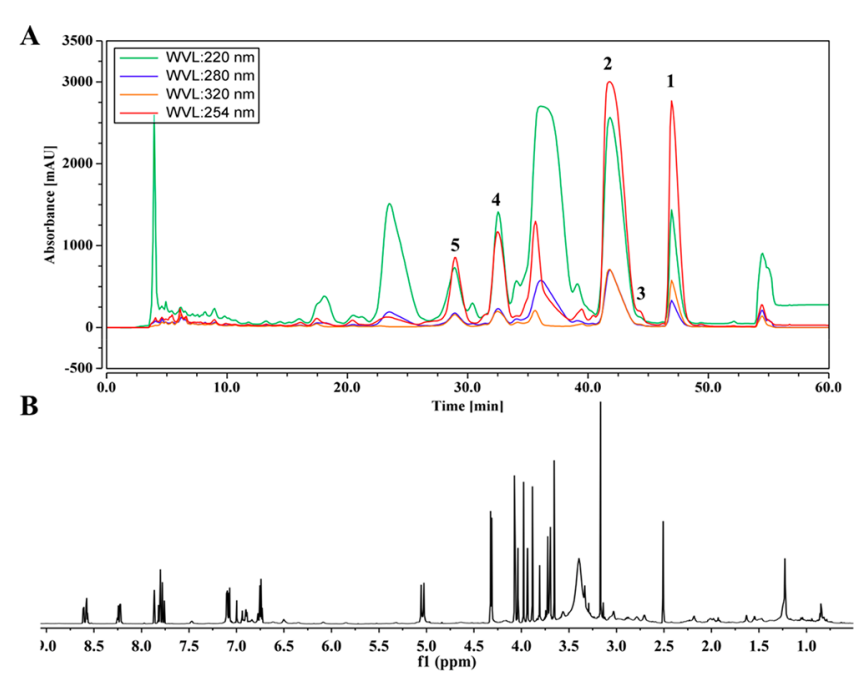

Figure 3. (A) HPLC profile of the alkaloid fraction of D. aromatica. (B) NMR fingerprint of the alkaloid fraction of D. aromatica.

NMR data (Tables 1 and 2) and 2D NMR experiments suggested that compounds $\mathbf{1 - 5}$ are closely related, all possessing a 6,7-dimethoxy-2-methylisoquinolinium moiety. ${ }^{1} \mathrm{H}$ and ${ }^{13} \mathrm{C}$ NMR spectra of compound 1 showed a number of paired signals, indicating a significant level of symmetry. The structure of compound 1 (Figure 4) was unambiguously identified as the TFA salt of the known compound phaeantharine, an alkaloid previously isolated from Phaeanthus ebracteolatus, by comparison of the observed and reported data. $^{18}$

Compound 2 (Figure 4) was isolated as a bright yellow solid, and the molecular formula for the free base of $\mathbf{2}$ was determined to be $\mathrm{C}_{39} \mathrm{H}_{43} \mathrm{~N}_{2} \mathrm{O}_{6}^{+}$by HRESIMS data on the [M $\left.-\mathrm{CF}_{3} \mathrm{COO}^{-}\right]^{+}$ion at $\mathrm{m} / z 635.3108$ (calcd for $\mathrm{C}_{39} \mathrm{H}_{43} \mathrm{~N}_{2} \mathrm{O}_{6}{ }^{+}$, 635.3116). In the ${ }^{13} \mathrm{C}$ NMR spectrum of 2 (Table 1 ), 39 carbon signals were observed, assigned as seven methyls, of which five are part of methoxy groups $\left(\delta_{\mathrm{C}} 55.1,55.9,56.3\right.$, 57.0, and 57.2), four methylenes, including one bearing a heteroatom $\left(\delta_{\mathrm{C}} 44.7\right)$, one aliphatic methine bearing heteroatoms $\left(\delta_{\mathrm{C}} 64.3\right)$, and 27 aromatic carbons arising from four aromatic rings. The ${ }^{1} \mathrm{H}$ NMR spectrum (Table 2) indicated the presence of one symmetrical para-disubstituted aromatic ring $\left[\delta_{\mathrm{H}} 6.77(2 \mathrm{H}, \mathrm{d}, J=8.7 \mathrm{~Hz}), 7.06(2 \mathrm{H}, \mathrm{d}, J=8.7\right.$ $\mathrm{Hz})]$, a 1,2,4-trisubstituted aromatic $\operatorname{ring}\left[\delta_{\mathrm{H}} 6.95(\mathrm{~d}, J=2.4\right.$ $\mathrm{Hz}), 7.11(\mathrm{~d}, J=8.9 \mathrm{~Hz}), 6.92(\mathrm{dd}, J=2.4,8.9 \mathrm{~Hz})]$, a $1,6,7-$ trisubstituted isoquinolinium moiety $\left[\delta_{\mathrm{H}} 8.53(\mathrm{~d}, J=7.2 \mathrm{~Hz})\right.$, $8.21(\mathrm{~d}, J=7.2 \mathrm{~Hz}), 7.73(\mathrm{~s}), 7.82(\mathrm{~s})]$, two aromatic proton singlets $\left[\delta_{\mathrm{H}} 6.85,5.71(\right.$ each $\left.1 \mathrm{H}, \mathrm{s})\right]$ due to a $1,2,4,5-$ tetrasubstituted aromatic ring, and seven heteroatom-bearing methyl singlets $\left[\delta_{\mathrm{H}} 2.84,3.29,3.69,3.74,3.93,4.04,4.30\right]$. 
Table 1. ${ }^{1} \mathrm{H}$ NMR Data of Compounds $1-5$ in DMSO- $d_{6}$ at $800 \mathrm{MHz}$

\begin{tabular}{|c|c|c|c|c|c|}
\hline & 1 & 2 & 3 & 4 & 5 \\
\hline position & $\begin{array}{c}\delta_{\mathrm{H}}, \text { mult. }(J \\
\text { in } \mathrm{Hz})\end{array}$ & $\begin{array}{l}\delta_{\mathrm{H}}, \text { mult. }(J \\
\text { in } \mathrm{Hz})\end{array}$ & $\begin{array}{c}\delta_{\mathrm{H}}, \text { mult. }(J \\
\text { in } \mathrm{Hz})\end{array}$ & $\begin{array}{c}\delta_{\mathrm{H}} \text {, mult. }(J \\
\text { in } \mathrm{Hz})\end{array}$ & $\begin{array}{l}\delta_{\mathrm{H}}, \text { mult. } \\
(J \text { in } \mathrm{Hz})\end{array}$ \\
\hline 3 & $\begin{array}{r}8.51, \mathrm{~d} \\
(7.0)\end{array}$ & $\begin{array}{r}8.53, \mathrm{~d} \\
(7.2)\end{array}$ & $\begin{array}{r}8.50, \mathrm{~d} \\
(6.8)\end{array}$ & $\begin{array}{r}8.53, \mathrm{~d} \\
(7.2)\end{array}$ & $\begin{array}{r}7.35, \mathrm{~d} \\
(7.2)\end{array}$ \\
\hline 4 & $\begin{array}{r}8.20, \mathrm{~d} \\
(6.8)\end{array}$ & $\begin{array}{r}8.21, \mathrm{~d} \\
(7.2)\end{array}$ & $\begin{array}{r}8.19, \mathrm{~d} \\
(6.7)\end{array}$ & $\begin{array}{r}8.21, \mathrm{~d} \\
(6.7)\end{array}$ & $\begin{array}{r}6.52, \mathrm{~d} \\
(7.2)\end{array}$ \\
\hline 5 & $7.73, \mathrm{~s}$ & $7.73, \mathrm{~s}$ & $7.73, \mathrm{~s}$ & $7.74, \mathrm{~s}$ & $7.15, \mathrm{~s}$ \\
\hline 8 & $7.78, \mathrm{~s}$ & $7.82, \mathrm{~s}$ & $7.79, \mathrm{~s}$ & $7.83, \mathrm{~s}$ & $7.58, \mathrm{~s}$ \\
\hline \multirow[t]{2}{*}{$\alpha$} & $4.98, \mathrm{~s}$ & $\begin{array}{l}5.04, \mathrm{~d} \\
(17.3)\end{array}$ & $4.93, \mathrm{~s}$ & $5.04, \mathrm{~s}$ & \\
\hline & & $\begin{array}{l}4.99, \mathrm{~d} \\
(17.3)\end{array}$ & & & \\
\hline 10 & $\begin{array}{r}6.98, \mathrm{~d} \\
(2.6)\end{array}$ & $\begin{array}{r}6.95, \mathrm{~d} \\
(2.4)\end{array}$ & $\begin{array}{r}6.91, \mathrm{~d} \\
(1.8)\end{array}$ & $\begin{array}{r}7.13, \mathrm{~d} \\
(2.1)\end{array}$ & \\
\hline 13 & $\begin{array}{r}7.07, \mathrm{~d} \\
(8.4)\end{array}$ & $\begin{array}{r}7.11, \mathrm{~d} \\
(8.9)\end{array}$ & $\begin{array}{r}6.88, \mathrm{~d} \\
(8.4)\end{array}$ & $\begin{array}{r}7.16, \mathrm{~d} \\
(8.8)\end{array}$ & \\
\hline 14 & $\begin{array}{l}6.89, \mathrm{dd} \\
\quad(2.1,8.4)\end{array}$ & $\begin{array}{l}6.92, \mathrm{dd} \\
\quad(2.4,8.9)\end{array}$ & $\begin{array}{l}6.74, \mathrm{dd} \\
\quad(2.3,6.8)\end{array}$ & $\begin{array}{l}\text { 7.00, dd } \\
\quad(2.1,8.6)\end{array}$ & \\
\hline $1^{\prime}$ & & $4.53 \mathrm{~m}$ & & & \\
\hline \multirow[t]{2}{*}{$3^{\prime}$} & $\begin{array}{r}8.53, \mathrm{~d} \\
(7.2)\end{array}$ & $3.73, \mathrm{~m}$ & $\begin{array}{r}8.54, \mathrm{~d} \\
(6.9)\end{array}$ & & \\
\hline & & $3.39, \mathrm{~m}$ & & & \\
\hline $4^{\prime}$ & $\begin{array}{r}8.22, \mathrm{~d} \\
(6.8)\end{array}$ & 2.99, m & $\begin{array}{r}8.22, \mathrm{~d} \\
(6.7)\end{array}$ & & \\
\hline $5^{\prime}$ & $7.76, \mathrm{~s}$ & $6.85, \mathrm{~s}$ & $7.76, \mathrm{~s}$ & & \\
\hline $8^{\prime}$ & $7.84, \mathrm{~s}$ & $5.71, \mathrm{~s}$ & $7.84, \mathrm{~s}$ & & \\
\hline$\alpha^{\prime}$ & $5.01, \mathrm{~s}$ & $\begin{array}{l}3.37, \mathrm{~m} \\
2.96, \mathrm{~m}\end{array}$ & $5.01, \mathrm{~s}$ & $9.89, \mathrm{~s}$ & \\
\hline $10^{\prime} / 14^{\prime}$ & $\begin{array}{l}7.07, \mathrm{dd} \\
\quad(5.2,8.4)\end{array}$ & $\begin{array}{r}7.06, \mathrm{~d} \\
(8.7)\end{array}$ & $\begin{array}{c}7.08, \mathrm{dd} \\
(8.8)\end{array}$ & $\begin{array}{r}7.86, \mathrm{~d} \\
(8.7)\end{array}$ & \\
\hline $11^{\prime} / 13^{\prime}$ & $\begin{array}{l}6.74, \mathrm{dd} \\
\quad(2.1,8.4)\end{array}$ & $\begin{array}{r}6.77, \mathrm{~d} \\
(8.7)\end{array}$ & $\begin{array}{l}6.75, \mathrm{dd} \\
\quad(1.7,8.7)\end{array}$ & $\begin{array}{r}6.95, \mathrm{~d} \\
(8.7)\end{array}$ & \\
\hline $1-\mathrm{OH}$ & & & & & 6.54 brs \\
\hline $\mathrm{CH}_{3} \mathrm{~N}-2$ & $4.27, \mathrm{~s}$ & $4.30, \mathrm{~s}$ & $4.28, \mathrm{~s}$ & $4.32, \mathrm{~s}$ & $4.32, \mathrm{~s}$ \\
\hline $\mathrm{CH}_{3} \mathrm{O}-6$ & $4.06, \mathrm{~s}$ & $4.04, \mathrm{~s}$ & $4.06, \mathrm{~s}$ & $4.07, \mathrm{~s}$ & $4.07, \mathrm{~s}$ \\
\hline $\mathrm{CH}_{3} \mathrm{O}-7$ & $3.87, \mathrm{~s}$ & $3.93, \mathrm{~s}$ & $3.88, \mathrm{~s}$ & $3.94, \mathrm{~s}$ & $3.94, \mathrm{~s}$ \\
\hline $12-\mathrm{OH}$ & & & $9.73, \mathrm{~s}$ & & \\
\hline $\mathrm{CH}_{3} \mathrm{O}-12$ & $3.64, \mathrm{~s}$ & $3.69, \mathrm{~s}$ & & $3.69, \mathrm{~s}$ & \\
\hline $\mathrm{CH}_{3} \mathrm{~N}-2^{\prime}$ & $4.28, \mathrm{~s}$ & $2.84, \mathrm{~s}$ & $4.29, \mathrm{~s}$ & & \\
\hline $\mathrm{CH}_{3} \mathrm{O}-6^{\prime}$ & $4.07, \mathrm{~s}$ & $3.74, \mathrm{~s}$ & $4.07, \mathrm{~s}$ & & \\
\hline $\mathrm{CH}_{3} \mathrm{O}-7^{\prime}$ & $3.96, \mathrm{~s}$ & $3.29, \mathrm{~s}$ & $3.96, \mathrm{~s}$ & & \\
\hline
\end{tabular}

These NMR spectroscopic features were closely related to those of phaeantharine (1). However, instead of the second unit of a 1,6,7-trisubstituted isoquinolinium moiety in 1, compound 2 showed a 1,2,4,5-tetrasubstituted aromatic ring, together with additional aliphatic methylenes $\left(\delta_{\mathrm{C}} 21.3,44.7\right)$ and methine $\left(\delta_{\mathrm{C}} 64.3\right)$ carbons, suggesting an aliphatic $\mathrm{B}^{\prime}$-ring.

The structures of the $\mathrm{A}, \mathrm{A}^{\prime}, \mathrm{B}, \mathrm{C}$, and $\mathrm{C}^{\prime}$ aromatic rings and their connections in compound $\mathbf{2}$ were determined by comparison to the observed data of phaeantharine (1). The structure of the $\mathrm{B}^{\prime}$-ring of $\mathbf{2}$ was determined from the HMBC correlations of $\mathrm{H}-1^{\prime}\left(\delta_{\mathrm{H}} 4.53, \mathrm{~m}, \mathrm{CH}\right)$ with C-8'a $\left(\delta_{\mathrm{C}} 121.3\right.$, C), $\mathrm{H}_{2}-3^{\prime}\left(\delta_{\mathrm{H}} 3.73,3.39, \mathrm{~m}, \mathrm{CH}_{2}\right)$ with C-1'/C-4'a, $\mathrm{H}_{2}-4^{\prime}\left(\delta_{\mathrm{H}}\right.$ $\left.2.99, \mathrm{~m}, \mathrm{CH}_{2}\right)$ with $\mathrm{C}-4^{\prime} \mathrm{a} / \mathrm{C}-8^{\prime} \mathrm{a}$, and the $\mathrm{CH}_{3} \mathrm{~N}\left(\delta_{\mathrm{H}} 2.84\right)$ group with $\delta_{\mathrm{C}} 64.3\left(\mathrm{CH}, \mathrm{C}-1^{\prime}\right)$ and $44.7\left(\mathrm{CH}_{2}, \mathrm{C}-3^{\prime}\right)$. Peaks of the aromatic proton at $\delta_{\mathrm{H}} 6.77\left(2 \mathrm{H}, \mathrm{H}-11^{\prime}, \mathrm{H}-13^{\prime}\right)$ with $\mathrm{C}-9^{\prime}$ $\left(\delta_{\mathrm{C}} 130.1\right) / \mathrm{C}-12^{\prime}\left(\delta_{\mathrm{C}} 157.2\right), 7.06\left(2 \mathrm{H}, \mathrm{H}-10^{\prime}, \mathrm{H}-14^{\prime}\right)$ with C$12^{\prime} / \mathrm{C}-\alpha^{\prime}\left(\delta_{\mathrm{C}} 40.2\right)$, both $\mathrm{H}_{2}-\alpha^{\prime}\left(\delta_{\mathrm{H}} 3.37,2.96, \mathrm{~m}, \mathrm{CH}_{2}\right)$ and $\mathrm{H}-1^{\prime}\left(\delta_{\mathrm{H}} 4.53\right)$ with $\mathrm{C}-8^{\prime}$ a, and $\mathrm{H}-\alpha^{\prime}$ with $\mathrm{C}-14^{\prime}\left(\delta_{\mathrm{C}} 131.7\right)$ confirmed the connection of the para-disubstituted aromatic $\mathrm{C}^{\prime}$-ring via another methylene group, $\mathrm{C}-\alpha^{\prime}$, to the heteroatombearing methine $\left(\delta_{\mathrm{C}} 64.3, \mathrm{C}-1^{\prime}\right)$, which was connected to C-
Table $2 .{ }^{13} \mathrm{C}$ NMR Data of Compounds $1-5$ in DMSO- $d_{6}$ at $200 \mathrm{MHz}$

\begin{tabular}{|c|c|c|c|c|c|}
\hline & 1 & 2 & 3 & 4 & 5 \\
\hline position & $\delta_{\mathrm{C}}$, type & $\delta_{\mathrm{C}}$, type & $\delta_{\mathrm{C}}$, type & $\delta_{\mathrm{C}}$, type & $\delta_{\mathrm{C}}$, type \\
\hline 1 & 155.2, C & 155.7, C & 156.0, C & 155.6, C & 161.2, C \\
\hline 3 & 136.2, CH & $136.8, \mathrm{CH}$ & 136.4, CH & $136.5, \mathrm{CH}$ & 132.4, CH \\
\hline 4 & 122.1, CH & $122.7, \mathrm{CH}$ & 122.3, CH & $122.4, \mathrm{CH}$ & $104.9, \mathrm{CH}$ \\
\hline $4 a$ & 135.6, C & 136.2, C & 136.2, C & 136.1, C & 132.9, C \\
\hline 5 & 106.4, CH & 106.9, CH & 106.6, CH & $106.8, \mathrm{CH}$ & $107.0, \mathrm{CH}$ \\
\hline 6 & 156.7, C & 157.2, C & 157.6, C & 157.3, C & 153.3, C \\
\hline 7 & 152.6, C & 153.2, C & 153.2, C & 153.1, C & 149.2, C \\
\hline 8 & 106.0, CH & 106.4, CH & 106.6, CH & 106.4, CH & $107.2, \mathrm{CH}$ \\
\hline $8 \mathrm{a}$ & 123.7, C & $124.2, \mathrm{C}$ & 124.1, C & $124.3, \mathrm{C}$ & $119.5, \mathrm{C}$ \\
\hline$\alpha$ & 33.0, $\mathrm{CH}_{2}$ & 33.5, $\mathrm{CH}_{2}$ & 33.1, $\mathrm{CH}_{2}$ & 33.5, $\mathrm{CH}_{2}$ & \\
\hline 9 & 127.8, C & 128.2, C & 126.7, C & 128.5, C & \\
\hline 10 & 122.0, CH & $122.3, \mathrm{CH}$ & $122.8, \mathrm{CH}$ & 123.0, $\mathrm{CH}$ & \\
\hline 11 & 143.3, C & 144.0, C & 142.6, C & 142.7, C & \\
\hline 12 & 150.4, C & 151.0, C & 149.0, C & 150.9, C & \\
\hline 13 & 114.0, $\mathrm{CH}$ & $114.5, \mathrm{CH}$ & 118.1, CH & $114.6, \mathrm{CH}$ & \\
\hline 14 & 125.4, CH & $125.8, \mathrm{CH}$ & 125.7, CH & 126.7, CH & \\
\hline $1^{\prime}$ & $155.4, \mathrm{C}$ & $64.3, \mathrm{CH}$ & 156.0, C & & \\
\hline $3^{\prime}$ & $136.3, \mathrm{CH}$ & 44.7, $\mathrm{CH}_{2}$ & $136.4, \mathrm{CH}$ & & \\
\hline $4^{\prime}$ & $122.2, \mathrm{CH}$ & 21.3, $\mathrm{CH}_{2}$ & $122.3, \mathrm{CH}$ & & \\
\hline $4 a^{\prime}$ & 135.7, C & $122.3, \mathrm{C}$ & $136.2, \mathrm{C}$ & & \\
\hline $5^{\prime}$ & 106.4, CH & $112.1, \mathrm{CH}$ & 106.6, CH & & \\
\hline $6^{\prime}$ & $156.8, \mathrm{C}$ & $148.9, \mathrm{C}$ & 157.6, C & & \\
\hline $7^{\prime}$ & 152.7, C & 146.9, C & 153.2, C & & \\
\hline $8^{\prime}$ & $105.9, \mathrm{CH}$ & 111.6, CH & $106.3, \mathrm{CH}$ & & \\
\hline $8 a^{\prime}$ & $123.8, \mathrm{C}$ & $121.3, \mathrm{C}$ & 124.1, C & & \\
\hline$\alpha^{\prime}$ & 33.2, $\mathrm{CH}_{2}$ & $40.2, \mathrm{CH}_{2}$ & $33.8, \mathrm{CH}_{2}$ & 191.6, CH & \\
\hline $9^{\prime}$ & $128.6, \mathrm{C}$ & $130.1, \mathrm{C}$ & 129.2, C & $131.3, \mathrm{C}$ & \\
\hline $10^{\prime} / 14^{\prime}$ & 129.6, CH & 131.7, CH & $129.8, \mathrm{CH}$ & 132.1, CH & \\
\hline $11^{\prime} / 13^{\prime}$ & 116.4, $\mathrm{CH}$ & $116.3, \mathrm{CH}$ & $116.8, \mathrm{CH}$ & $116.2, \mathrm{CH}$ & \\
\hline $12^{\prime}$ & $156.7, \mathrm{C}$ & $157.2, \mathrm{C}$ & $157.4, \mathrm{C}$ & $163.2, \mathrm{C}$ & \\
\hline $\mathrm{CH}_{3} \mathrm{~N}-2$ & $45.8, \mathrm{CH}_{3}$ & $46.4, \mathrm{CH}_{3}$ & 46.1, $\mathrm{CH}_{3}$ & 46.3, $\mathrm{CH}_{3}$ & 46.3, $\mathrm{CH}_{3}$ \\
\hline $\mathrm{CH}_{3} \mathrm{O}-6$ & 56.7, $\mathrm{CH}_{3}$ & $57.2, \mathrm{CH}_{3}$ & $57.2, \mathrm{CH}_{3}$ & $57.2, \mathrm{CH}_{3}$ & 57.2, $\mathrm{CH}_{3}$ \\
\hline $\mathrm{CH}_{3} \mathrm{O}-7$ & $56.8, \mathrm{CH}_{3}$ & 57.0, $\mathrm{CH}_{3}$ & $56.9, \mathrm{CH}_{3}$ & $56.9, \mathrm{CH}_{3}$ & $56.9, \mathrm{CH}_{3}$ \\
\hline $\mathrm{CH}_{3} \mathrm{O}-12$ & 55.7, $\mathrm{CH}_{3}$ & $56.3, \mathrm{CH}_{3}$ & & $56.2, \mathrm{CH}_{3}$ & \\
\hline $\mathrm{CH}_{3} \mathrm{~N}-2^{\prime}$ & $45.9, \mathrm{CH}_{3}$ & $39.9, \mathrm{CH}_{3}$ & 46.1, $\mathrm{CH}_{3}$ & & \\
\hline $\mathrm{CH}_{3} \mathrm{O}-6^{\prime}$ & $56.8, \mathrm{CH}_{3}$ & $55.9, \mathrm{CH}_{3}$ & 57.2, $\mathrm{CH}_{3}$ & & \\
\hline $\mathrm{CH}_{3} \mathrm{O}-7^{\prime}$ & $56.6, \mathrm{CH}_{3}$ & 55.1, $\mathrm{CH}_{3}$ & $56.9, \mathrm{CH}_{3}$ & & \\
\hline
\end{tabular}

$8^{\prime}$ a of the $\mathrm{A}^{\prime}$-ring. HMBC correlations of the methoxy protons $\left(\delta_{\mathrm{H}} 4.04, \mathrm{~s}, \mathrm{CH}_{3} \mathrm{O}-6,3.93, \mathrm{~s}, \mathrm{CH}_{3} \mathrm{O}-7,3.69, \mathrm{~s}, \mathrm{CH}_{3} \mathrm{O}-12\right.$, 3.74, s, $\mathrm{CH}_{3} \mathrm{O}-6^{\prime}$, and $\left.3.29, \mathrm{~s}, \mathrm{CH}_{3} \mathrm{O}-7^{\prime}\right)$ with deshielded C-6 $\left(\delta_{\mathrm{C}}\right.$ $157.2), \mathrm{C}-7\left(\delta_{\mathrm{C}} 153.2\right), \mathrm{C}-12\left(\delta_{\mathrm{C}} 151.0\right), \mathrm{C}-6^{\prime}\left(\delta_{\mathrm{C}} 148.9\right)$, and $\mathrm{C}-7^{\prime}\left(\delta_{\mathrm{C}} 146.9\right)$, respectively, provided the $2 \mathrm{D}$ structure of compound 2.

The absolute configuration of the C-1' stereogenic center of 2 was established by the electronic circular dichroism (ECD) spectrum, which exhibited Cotton effects similar to the calculated spectrum of the $1^{\prime} R-2$ diastereoisomer (Figure 6). On the basis of the above evidence, the structure of compound 2 was elucidated as a new compound, named $(R)$ nomimantharine trifluoroacetate.

Compound 3 (Figure 4) was isolated as a bright yellow solid, and the HRESIMS data showed a molecular ion at $\mathrm{m} / z$ $617.2641\left[\mathrm{M}-2 \mathrm{CF}_{3} \mathrm{COO}^{-}-\mathrm{H}\right]^{+}\left(\right.$calcd for $\mathrm{C}_{38} \mathrm{H}_{37} \mathrm{~N}_{2} \mathrm{O}_{6}^{+}$, 617.2646), which was consistent with a molecular formula for the free base of 3 as $\mathrm{C}_{38} \mathrm{H}_{38} \mathrm{~N}_{2} \mathrm{O}_{6}{ }^{2+}, 14$ Da less than that of $\mathbf{1}$. The UV spectrum was similar to those of $\mathbf{1}$ and 2 . A comparison of the ${ }^{1} \mathrm{H}$ and ${ }^{13} \mathrm{C}$ NMR spectroscopic data (Table 


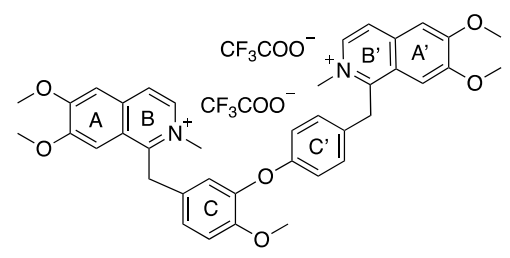

1

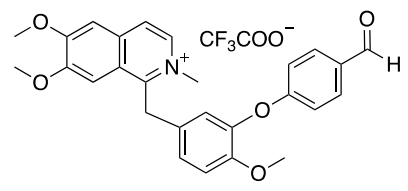

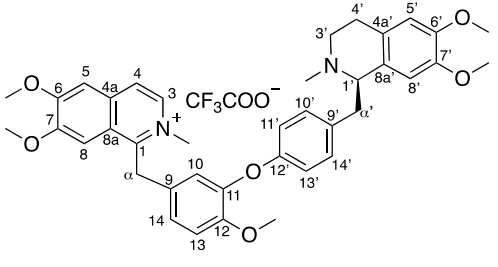

2

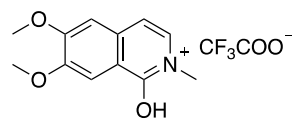

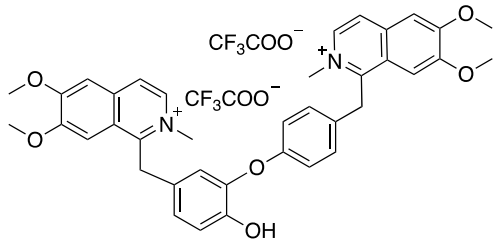

3

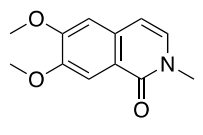

6

Figure 4. Structures of compounds 1-6.

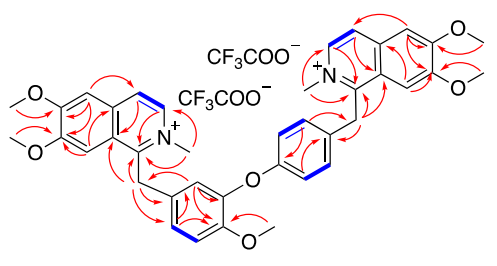

1

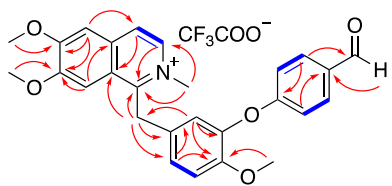

4

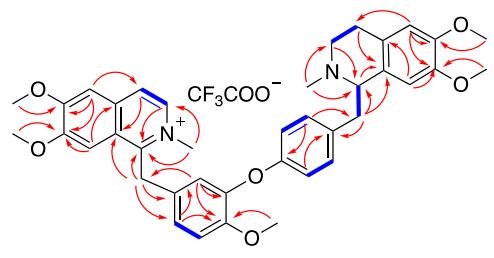

2

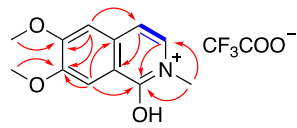

5

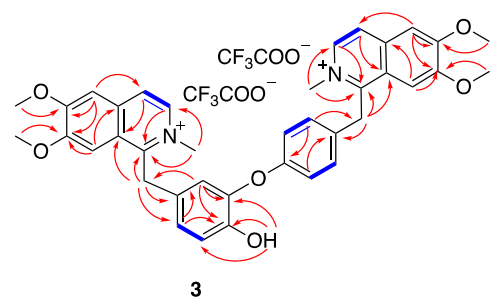

- COSY

HMBC

Figure 5. COSY and key HMBC correlations of compounds 1-5.

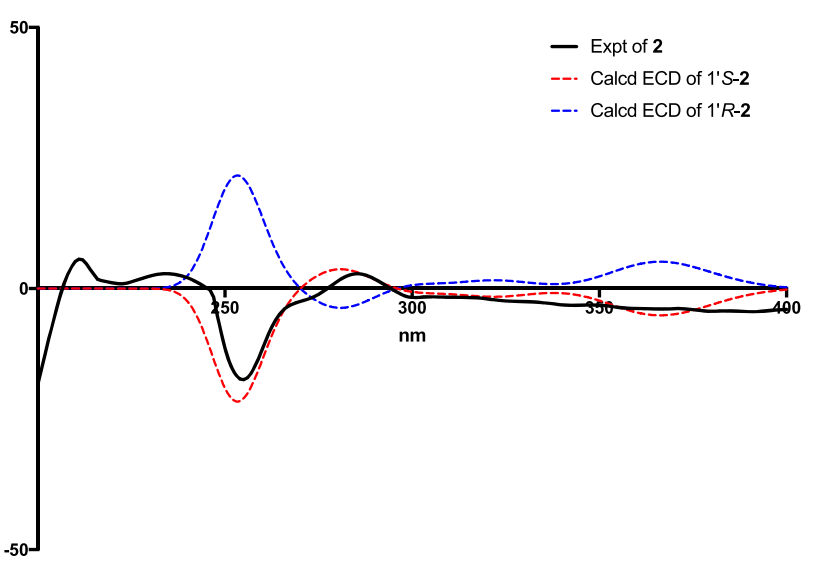

Figure 6. Experimental and computed ECD spectra of 2.

1) with those of $\mathbf{1}$ showed that the only difference was the absence of one methoxy group in the C-ring of 3 . In the ${ }^{1} \mathrm{H}$ NMR spectrum of 3, four methoxy proton singlets were observed $\left(\delta_{\mathrm{H}} 4.06, \mathrm{CH}_{3} \mathrm{O}-6,3.88, \mathrm{CH}_{3} \mathrm{O}-7,4.07, \mathrm{CH}_{3} \mathrm{O}-66^{\prime}\right.$, 3.96, $\left.\mathrm{CH}_{3} \mathrm{O}-7^{\prime}\right)$ with a phenolic proton at $\delta_{\mathrm{H}} 9.73$. $\mathrm{HMBC}$ correlations (Figure 5$)$ from $\delta_{\mathrm{H}} 9.73$ to C-11 $\left(\delta_{\mathrm{C}} 142.6, \mathrm{C}\right) / \mathrm{C}$ $12\left(\delta_{\mathrm{C}} 149.0, \mathrm{C}\right) / \mathrm{C}-13\left(\delta_{\mathrm{C}} 118.1, \mathrm{CH}\right)$ supported the connection of the hydroxy group to C-12. Finally, compound
3 was identified as a new compound, named 12-de-Omethylphaeantharine trifluoroacetate.

Compound 4 (Figure 4) was isolated as a yellow solid, and the HRESIMS data showed a molecular ion at $\mathrm{m} / \mathrm{z} 444.1800$ $\left[\mathrm{M}-\mathrm{CF}_{3} \mathrm{COO}^{-}\right]^{+}$(calcd for $\mathrm{C}_{27} \mathrm{H}_{26} \mathrm{NO}_{5}{ }^{+}, 444.1805$ ), which was consistent with a molecular formula for the free base of 4 as $\mathrm{C}_{27} \mathrm{H}_{26} \mathrm{NO}_{5}{ }^{+}$. Analysis of its ${ }^{1} \mathrm{H}$ and ${ }^{13} \mathrm{C}$ NMR spectroscopic data suggested that instead of having two symmetrical units as compounds 1-3, there was only one benzyltetrahydroisoquinoline unit present in compound 4. This was consistent with the molecular formula, accommodating one nitrogen atom. The ${ }^{1} \mathrm{H} \mathrm{NMR}$ and ${ }^{13} \mathrm{C}$ NMR spectroscopic data of rings A, B, C, and $\mathrm{C}^{\prime}$ were identical to those of $\mathbf{1}$ and $\mathbf{2}$ (Tables 1 , 2). A significantly deshielded chemical shift at $\delta_{\mathrm{C}} 191.6$ was observed in 4, suggesting a formyl proton resonating at $\delta_{\mathrm{H}} 9.89$ by HSQC data. The formyl group was assigned at C- $\alpha^{\prime}$ due to the HMBC correlations from $\delta_{\mathrm{H}} 9.89$ to $\mathrm{C}-10^{\prime} / \mathrm{C}-14^{\prime}\left(\delta_{\mathrm{C}}\right.$ $132.1, \mathrm{CH})$ and from $\mathrm{H}-10^{\prime} / \mathrm{H}-14^{\prime}\left(\delta_{\mathrm{H}} 7.86, \mathrm{~d}, J=8.7 \mathrm{~Hz}\right)$ to $\delta_{\mathrm{C}}$ 191.6. Therefore, compound 4 was determined to be nominanthranal trifluoroacetate.

Compound 5 (Figure 4) was obtained as a white solid and gave a molecular formula for the free base as $\mathrm{C}_{12} \mathrm{H}_{14} \mathrm{NO}_{3}{ }^{+}$, as deduced from the positive HRESIMS $(\mathrm{m} / z 220.0966[\mathrm{M}-$ $\left.\mathrm{CF}_{3} \mathrm{COO}^{-}\right]^{+}$, calcd for $\left.\mathrm{C}_{12} \mathrm{H}_{14} \mathrm{NO}_{3}^{+}, 220.0968\right)$. The ${ }^{1} \mathrm{H}$ and ${ }^{13} \mathrm{C}$ NMR data of 5 suggested it possessed a similar 6,7dimethoxy-2-methylisoquinolinium moiety to compounds 1- 
4. The detected nine aromatic carbons were assigned to the isoquinolinium moiety based on comparison with data of compounds 1-4. Three heteroatom-bearing methyl singlets $\left(\delta_{\mathrm{H}} 4.32,4.07,3.94\right)$ were assigned to $\mathrm{CH}_{3} \mathrm{~N}-2, \mathrm{CH}_{3} \mathrm{O}-6$, and $\mathrm{CH}_{3} \mathrm{O}-7$ on the basis of their HMBC correlations with C-1 $\left(\delta_{\mathrm{C}}\right.$ $161.2, \mathrm{C}) / \mathrm{C}-3\left(\delta_{\mathrm{C}} 132.4, \mathrm{CH}\right), \mathrm{C}-6\left(\delta_{\mathrm{C}} 153.3, \mathrm{C}\right)$, and C-7 $\left(\delta_{\mathrm{C}} 149.2, \mathrm{C}\right)$, respectively. Comparison of the molecular formula suggested the presence of a hydroxy group, which was detected as a broad signal at $\delta_{\mathrm{H}} 6.54$. The deshielded chemical shift of C-1 $\left(\delta_{\mathrm{C}} 161.2, \mathrm{C}\right)$ suggested its connection with the hydroxy group. Consequently, 5 was deduced as the trifluoroacetate salt of the enolic form of $N$-methyl-6,7dimethoxyisoquinolone (6). ${ }^{19}$

Compounds $\mathbf{1 - 5}$ were subjected to in vitro screening against Mycobacteria strains using the $M$. smegmatis strain $\mathrm{mc}^{2} 155$, M. tuberculosis $\mathrm{mc}^{2} 6220$ biosafety level 2 (BSL2) strain, and the wild-type strain H37Rv (Table 3). (R)-

Table 3. In Vitro Antimicrobial Activity of the Isolated Compounds

\begin{tabular}{|c|c|c|c|c|c|}
\hline strain & 1 & 2 & 3 & 4 & 5 \\
\hline M. smegmatis $\mathrm{MIC}(\mu \mathrm{M})$ & 158.2 & 39.4 & $>100$ & $>100$ & $>100$ \\
\hline $\begin{array}{l}\text { Mtb mc } 6220 \text { BSL2 } \\
\text { growth inhibition (\%) }\end{array}$ & 0 & $\begin{array}{l}70 \text { at } \\
6.25 \mu \mathrm{M}\end{array}$ & $\mathrm{NT}^{a}$ & NT & NT \\
\hline $\begin{array}{l}\text { Mtb } \mathrm{mc}^{2} 6220 \text { BSL2MIC } \\
(\mu \mathrm{M})\end{array}$ & NT & 12.5 & NT & NT & NT \\
\hline Mtb H37Rv MIC $(\mu \mathrm{M})$ & NT & $>100$ & NT & NT & NT \\
\hline E. coli $\mathrm{MIC}(\mu \mathrm{M})$ & 39.6 & 157.5 & $>100$ & $>100$ & $>100$ \\
\hline $\begin{array}{l}\text { Staphylococcus aureus } \\
\text { MIC }(\mu \mathrm{M})\end{array}$ & 9.9 & 39.4 & $>100$ & $>100$ & $>100$ \\
\hline \multirow{5}{*}{$\begin{array}{l}\text { MRSA MIC } \\
(\mu \mathrm{M})\end{array}$} & 9.9 & 39.4 & NT & NT & NT \\
\hline & 39.6 & 39.4 & NT & NT & NT \\
\hline & 39.6 & 19.7 & NT & NT & NT \\
\hline & 9.9 & 39.4 & NT & NT & NT \\
\hline & 9.9 & 19.7 & NT & NT & NT \\
\hline
\end{tabular}

${ }^{a} \mathrm{NT}$ : not tested.

Nomimantharine trifluoroacetate (2) showed moderate activities against the $M$. smegmatis strain $\mathrm{mc}^{2} 155$ and $M t b$ $\mathrm{mc}^{2} 6220 \mathrm{BSL} 2$ strain with MIC values of 39.4 and $12.5 \mu \mathrm{M}$, respectively. Phaeantharine trifluoroacetate (1) displayed weak activity against the $M$. smegmatis strain $\mathrm{mc}^{2} 155$, but no inhibition was observed in the $M t b$ screens. Compounds 3-5 showed no activity (MIC > $100 \mu \mathrm{g} / \mathrm{mL}$ ) against the $M$. smegmatis strain $\mathrm{mc}^{2} 155$ and were, therefore, not evaluated in $M t b$ assays. Comparisons of the MIC values obtained for these compounds suggested that saturation of the $\mathrm{B}^{\prime}$-ring increases antitubercular activity.

Compounds $\mathbf{1 - 5}$ were evaluated for antimicrobial activity against E. coli and S. aureus. Phaeantharine trifluoroacetate (1) exhibited promising activity against $E$. coli and $S$. aureus with MIC values of 39.6 and $9.9 \mu \mathrm{M}$, respectively. $(R)$ Nomimantharine trifluoroacetate (2) showed weak activity in both assays with MIC values of 157.5 and $39.4 \mu \mathrm{M}$, respectively, whereas compounds 3-5 were inactive (MIC > $100 \mu \mathrm{g} / \mathrm{mL}$ ). In order to further evaluate the antibacterial potential of phaeantharine trifluoroacetate $(1)$ and $(R)$ nomimantharine trifluoroacetate (2) against drug-resistant $S$. aureus strains, their activities were measured against five clinical isolates of oxacillin/methicillin-resistant $S$. aureus (MRSA) (Table 3). Both compounds showed similar or even stronger levels of activity against the resistant isolates, indicating their potential as anti-MRSA agents.

\section{EXPERIMENTAL SECTION}

General Experimental Procedures. Optical rotations were recorded on a JASCO P-1020 polarimeter $(10 \mathrm{~cm}$ cell $)$. NMR spectra were recorded in DMSO- $d_{6}\left(\delta_{\mathrm{H}} 2.50\right.$ and $\left.\delta_{\mathrm{C}} 39.5\right)$ at $25^{\circ} \mathrm{C}$ on a Bruker Avance HDX $800 \mathrm{MHz}$ spectrometer equipped with a TCI cryoprobe. HRMS data were recorded on a Bruker maXis II ETD ESIqTOF. An Edwards Instrument Company Bioline orbital shaker was used for extraction. The HPLC system for fractionation for initial Mtb screening was a Waters 600 pump (Milford, MA, USA) fitted with a 996-photodiode array detector (PDA) and Gilson FC204 fraction collector (Middleton, WI, USA). Semipreparative HPLC was performed on a Thermo Ultimate 3000 with a PDA detector. A Phenomenex $\mathrm{C}_{18}$ Monolithic column $(5 \mu \mathrm{m}, 4.6 \times 100 \mathrm{~mm})$ was used for analytical HPLC; two Thermo Hypersil Gold $\mathrm{C}_{18}$ columns $(5 \mu \mathrm{m}$, $21.2 \times 250 \mathrm{~mm}$ and $5 \mu \mathrm{m}, 10 \times 250 \mathrm{~mm}$ ) were used for semipreparative HPLC. All solvents used for extraction, chromatography, $[\alpha]_{\mathrm{D}}$, and MS were HPLC grade, and $\mathrm{H}_{2} \mathrm{O}$ was Millipore MilliQ PF filtered.

Plant Material. The sample of D. aromatica NB024954 was collected and identified by P.I.F. in April 1999 from East of Rossville in north Queensland. A voucher specimen is lodged in the Queensland Herbarium (BRI-AQ606149).

Extraction and Purification of Compounds 1-5. The ground and freeze-dried $D$. aromatica $(17 \mathrm{~g})$ was extracted with $95 \%$ ethanol $(3 \times 300 \mathrm{~mL})$ overnight at room temperature $(\mathrm{rt})$ to afford a crude extract $(2.75 \mathrm{~g})$. The crude extract was fractionated using a Sephadex LH-20 column. Five fractions (I-V) were collected by eluting with $100 \% \mathrm{MeOH}$. Fraction II, containing the alkaloids, was chromatographed by HPLC (gradient from $60 \% \mathrm{H}_{2} \mathrm{O} / 40 \% \mathrm{MeOH}$ with $0.1 \%$ TFA to $20 \% \mathrm{H}_{2} \mathrm{O} / 80 \% \mathrm{MeOH}$ with $0.1 \%$ TFA, $9 \mathrm{~mL} / \mathrm{min}$ ) using a semipreparative reversed-phase Thermo Hypersil Gold $\mathrm{C}_{18}$ column (5 $\mu \mathrm{m}, 21.2 \times 250 \mathrm{~mm}$ ), leading to the isolation of phaeantharine trifluoroacetate (1) (retention time: $16.5 \mathrm{~min}, 12.6 \mathrm{mg}$ ), $(R)$ nomimantharine trifluoroacetate (2) (retention time: $15.6 \mathrm{~min}, 5.7$ $\mathrm{mg}$ ), and four subfractions, A-D. Subfraction B was further chromatographed by HPLC (isocratic $27 \% \mathrm{H}_{2} \mathrm{O} / \mathrm{MeOH}$ with $0.1 \%$ TFA, $4 \mathrm{~mL} / \mathrm{min}$ ) using a semipreparative reversed-phase Thermo Hypersil Gold $\mathrm{C}_{18}$ column $(5 \mu \mathrm{m}, 10 \times 250 \mathrm{~mm})$, leading to the isolation of 12-de-O-methylphaeantharine trifluoroacetate (3) (retention time: $98.8 \mathrm{~min}, 1.2 \mathrm{mg}$ ). Subfraction $\mathrm{C}$ was chromatographed by the same Thermo Hypersil Gold $\mathrm{C}_{18}$ column $(5 \mu \mathrm{m}, 10 \times 250$ $\mathrm{mm}$ ) eluting at a flow rate of $4 \mathrm{~mL} / \mathrm{min}$ with $35 \% \mathrm{H}_{2} \mathrm{O} / \mathrm{MeOH}$ with $0.1 \%$ TFA to yield nominanthranal trifluoroacetate (4) (retention time: $44.9 \mathrm{~min}, 2.1 \mathrm{mg}$ ) and the enolic form of 1-hydroxy-6,7dimethoxy-2-methylisoquinoline trifluoroacetate (5) (retention time: $41.2 \mathrm{~min}, 1.7 \mathrm{mg}$ ).

(R)-Nomimantharine trifluoroacetate (2): bright yellow solid; $[\alpha]_{\mathrm{D}}^{25}-147(c \quad 0.03, \mathrm{MeOH}) ; \mathrm{UV}(\mathrm{MeOH}) \lambda_{\max }(\log \varepsilon) 209 \mathrm{~nm}$ (3.42), $228 \mathrm{~nm}$ (2.67), $256 \mathrm{~nm}$ (3.39), $282 \mathrm{~nm}(0.63), 316 \mathrm{~nm}$ (0.62); ${ }^{1} \mathrm{H}$ NMR $\left(800 \mathrm{MHz}, \mathrm{DMSO}-d_{6}\right)$ and ${ }^{13} \mathrm{C}$ NMR data $(200$ MHz, DMSO- $d_{6}$ ), Tables 1 and 2; (+)-HRESIMS $m / z 635.3108$ [M $\left.-\mathrm{CF}_{3} \mathrm{COO}^{-}\right]^{+}$(calcd for $\left.\mathrm{C}_{39} \mathrm{H}_{43} \mathrm{~N}_{2} \mathrm{O}_{6}^{+}, 635.3116\right)$.

12-De-O-methylphaeantharine trifluoroacetate (3): bright yellow solid; UV (MeOH) $\lambda_{\max }(\log \varepsilon) 199 \mathrm{~nm}(2.60), 255 \mathrm{~nm}$ (2.92), 316 $\mathrm{nm}(0.53) ;{ }^{1} \mathrm{H}$ NMR $\left(800 \mathrm{MHz}\right.$, DMSO- $\left.d_{6}\right)$ and ${ }^{13} \mathrm{C}$ NMR data $(200$ $\mathrm{MHz}$, DMSO- $d_{6}$ ), Tables 1 and 2; (+)-HRESIMS $m / z$ 309.1357 [M $\left.-2 \mathrm{CF}_{3} \mathrm{COO}^{-}\right]^{2+}$ (calcd for $\left.\mathrm{C}_{38} \mathrm{H}_{38} \mathrm{~N}_{2} \mathrm{O}_{6}^{2+}, 309.1359\right), 617.2641[\mathrm{M}$ $\left.-2 \mathrm{CF}_{3} \mathrm{COO}^{-}-\mathrm{H}\right]^{+}$(calcd for $\left.\mathrm{C}_{38} \mathrm{H}_{37} \mathrm{~N}_{2} \mathrm{O}_{6}{ }^{+}, 617.2646\right)$.

Nominanthranal trifluoroacetate (4): yellow solid; UV (MeOH) $\lambda_{\max }(\log \varepsilon) 204 \mathrm{~nm}(3.20), 227 \mathrm{~nm}(1.76), 256 \mathrm{~nm}(2.36) ;{ }^{1} \mathrm{H}$ NMR $\left(800 \mathrm{MHz}, \mathrm{DMSO}-d_{6}\right)$ and ${ }^{13} \mathrm{C}$ NMR data $\left(200 \mathrm{MHz}, \mathrm{DMSO}-d_{6}\right)$, Tables 1 and 2; (+)-HRESIMS $\mathrm{m} / z$ 444.1800 $\left[\mathrm{M}-\mathrm{CF}_{3} \mathrm{COO}^{-}\right]^{+}$ (calcd for $\mathrm{C}_{27} \mathrm{H}_{26} \mathrm{NO}_{5}^{+}$, 444.1805).

1-Hydroxy-6,7-dimethoxy-2-methylisoquinoline trifluoroacetate (5): white solid; UV (MeOH) $\lambda_{\max }(\log \varepsilon) 205 \mathrm{~nm}(1.43), 226 \mathrm{~nm}$ (0.98), $255 \mathrm{~nm}(2.05) ;{ }^{1} \mathrm{H}$ NMR (800 MHz, DMSO-d $\left.d_{6}\right)$ and ${ }^{13} \mathrm{C}$ NMR data $\left(200 \mathrm{MHz}\right.$, DMSO- $\left.d_{6}\right)$, Tables 1 and 2; (+)-HRESIMS $\mathrm{m} /$ $z 220.0966\left[\mathrm{M}-2 \mathrm{CF}_{3} \mathrm{COO}^{-}\right]^{+}$(calcd for $\left.\mathrm{C}_{12} \mathrm{H}_{14} \mathrm{NO}_{3}{ }^{+}, 220.0968\right)$. 
DFT Calculations. The calculations were performed by using the density functional theory (DFT) as carried out in the Gaussian 03 program. The preliminary conformational distribution search was performed by HyperChem Release 8.0 software. GaussView 5.0 was used to view the conformational structures and change the input file for calculation. All ground-state geometries were optimized at the B3LYP/6-31G(d) level. Conformers within a $2 \mathrm{kcal} / \mathrm{mol}$ energy threshold from the global minimum were selected to calculate the electronic transitions. The overall calculated ECD spectra were obtained according to the Boltzmann weighting of each conformer.

Biological Assays. Compounds were evaluated for their antimycobacterial activities against $M$. smegmatis strain $\mathrm{mc}^{2} 155$ (ATCC 70084), ${ }^{20}$ M. tuberculosis $\mathrm{mc}^{2} 6220$ biosafety level 2 (BSL2) strain, and M. tuberculosis H37Rv. ${ }^{21}$ All the experiments were performed in triplicate.

Antimicrobial assays were performed according to the previously reported method. ${ }^{22}$

\section{ASSOCIATED CONTENT}

\section{s) Supporting Information}

The Supporting Information is available free of charge at https://pubs.acs.org/doi/10.1021/acs.jnatprod.0c01093.

Spectroscopic data of phaeantharine trifluoroacetate (1), $1 \mathrm{D}$ and 2D NMR and MS data of compounds 1-5, stable conformers of $R$-nomimantharine trifluoroacetate (2) (PDF)

\section{AUTHOR INFORMATION}

\section{Corresponding Authors}

Ronald J. Quinn - Griffith Institute for Drug Discovery, Griffith University, Brisbane, QLD 4111, Australia; (1) orcid.org/0000-0002-4022-2623; Phone: +61 73735 6006; Email: r.quinn@griffith.edu.au

Miaomiao Liu - Griffith Institute for Drug Discovery, Griffith University, Brisbane, QLD 4111, Australia; (1) orcid.org/ 0000-0003-0930-3617; Phone: +61 73735 6077; Email: miaomiao.liu@griffith.edu

\section{Authors}

Jianying Han - Griffith Institute for Drug Discovery, Griffith University, Brisbane, QLD 4111, Australia

Yunjiang Feng - Griffith Institute for Drug Discovery, Griffith University, Brisbane, QLD 4111, Australia

Gordon Guymer - Queensland Herbarium, Department of Environment and Science, Brisbane Botanic Gardens, Brisbane, QLD 4066, Australia

Paul I. Forster - Queensland Herbarium, Department of Environment and Science, Brisbane Botanic Gardens, Brisbane, QLD 4066, Australia

Complete contact information is available at:

https://pubs.acs.org/10.1021/acs.jnatprod.0c01093

\section{Notes}

The authors declare no competing financial interest.

\section{ACKNOWLEDGMENTS}

This research was funded by the Bill and Melinda Gates Foundation (OPP1035218, OPP1174957) with additional support from the Australian Research Council Discovery and Linkage Projects (DP160101429, LP120100485, LE120100170 (equipment), LE140100119 (equipment)). We acknowledge the NatureBank biota repository that is housed at the Griffith Institute for Drug Discovery at Griffith University (www.griffith.edu.au/gridd). We thank V. Ram- achandran, S. Sharma, S. Guptha, and S. DeSousa (formerly at AstraZeneca Bangalore) for the M. tuberculosis $\mathrm{H} 37 \mathrm{Rv}$ assay. We thank C. F. Nathan, R. Bryk, and Y. Ling (Abby and Howard P. Milstein Program in Chemical Biology and Translational Medicine, Weill Cornell Medical College) for the M. tuberculosis assays on the compounds.

\section{DEDICATION}

Dedicated to Dr. A. Douglas Kinghorn, The Ohio State University, for his pioneering work on bioactive natural products.

\section{REFERENCES}

(1) Hiramatsu, K.; Katayama, Y.; Matsuo, M.; Sasaki, T.; Morimoto, Y.; Sekiguchi, A.; Baba, T. J. Infect. Chemother. 2014, 20, 593-601.

(2) Schon, T.; Miotto, P.; Koser, C. U.; Viveiros, M.; Bottger, E.; Cambau, E. Clin. Microbiol. Infect. 2017, 23, 154-160.

(3) Liu, M. M.; El-Hossary, E. M.; Oelschlaeger, T. A.; Donia, M. S.; Quinn, R. J.; Abdelmohsen, U. R. Lancet Infect. Dis. 2019, 19, E237E245.

(4) Newman, D. J.; Cragg, G. M. J. Nat. Prod. 2020, 83, 770-803.

(5) Han, J.; Liu, M.; Jenkins, I. D.; Liu, X.; Zhang, L.; Quinn, R. J.; Feng, Y. Mar. Drugs 2020, 18, 352.

(6) Han, J. Y.; Zhang, J. Y.; Song, Z. J.; Liu, M. M.; Hu, J. S.; Hou, C. J.; Zhu, G. L.; Jiang, L.; Xia, X. K.; Quinn, R. J.; Feng, Y. J.; Zhang, L. X.; Hsiang, T.; Liu, X. T. Appl. Microbiol. Biotechnol. 2019, 103, $5167-5181$

(7) Harvey, A. L.; Edrada-Ebel, R.; Quinn, R. J. Nat. Rev. Drug Discovery 2015, 14, 111-129.

(8) Liu, M. M.; Abdel-Mageed, W. M.; Ren, B.; He, W. N.; Huang, P.; Li, X. L.; Bolla, K.; Guo, H.; Chen, C. X.; Song, F. H.; Dai, H. Q.; Quinn, R. J.; Grkovic, T.; Zhang, X. P.; Liu, X. T.; Zhang, L. X. Appl. Microbiol. Biotechnol. 2014, 98, 1077-1085.

(9) Han, J. Y.; Zhang, J. Y.; Song, Z. J.; Zhu, G. L.; Liu, M. M.; Dai, H. Q.; Hsiang, T.; Liu, X. T.; Zhang, L. X.; Quinn, R. J.; Feng, Y. J. Appl. Microbiol. Biotechnol. 2020, 104, 3835-3846.

(10) Camp, D.; Davis, R. A.; Campitelli, M.; Ebdon, J.; Quinn, R. J. J. Nat. Prod. 2012, 75, 72-81.

(11) Quinn, R. J.; Carroll, A. R.; Pham, N. B.; Baron, P.; Palframan, M. E.; Suraweera, L.; Pierens, G. K.; Muresan, S. J. Nat. Prod. 2008, 71, 464-468.

(12) McAlpine, J. B.; Chen, S. N.; Kutateladze, A.; MacMillan, J. B.; Appendino, G.; Barison, A.; Beniddir, M. A.; Biavatti, M. W.; Bluml, S.; Boufridi, A.; Butler, M. S.; Capon, R. J.; Choi, Y. H.; Coppage, D.; Crews, P.; Crimmins, M. T.; Csete, M.; Dewapriya, P.; Egan, J. M.; Garson, M. J.; Genta-Jouve, G.; Gerwick, W. H.; Gross, H.; Harper, M. K.; Hermanto, P.; Hook, J. M.; Hunter, L.; Jeannerat, D.; Ji, N. Y.; Johnson, T. A.; Kingston, D. G. I.; Koshino, H.; Lee, H. W.; Lewin, G.; Li, J.; Linington, R. G.; Liu, M. M.; McPhail, K. L.; Molinski, T. F.; Moore, B. S.; Nam, J. W.; Neupane, R. P.; Niemitz, M.; Nuzillard, J. M.; Oberlies, N. H.; Ocampos, F. M. M.; Pan, G.; Quinn, R. J.; Reddy, D. S.; Renault, J. H.; Rivera-Chavez, J.; Robien, W.; Saunders, C. M.; Schmidt, T. J.; Seger, C.; Shen, B.; Steinbeck, C.; Stuppner, H.; Sturm, S.; Taglialatela-Scafati, O.; Tantillo, D. J.; Verpoorte, R.; Wang, B. G.; Williams, C. M.; Williams, P. G.; Wist, J.; Yue, J. M.; Zhang, C.; Xu, Z. R.; Simmler, C.; Lankin, D. C.; Bisson, J.; Pauli, G. F. Nat. Prod. Rep. 2019, 36, 35-107.

(13) Renner, S. S.; Strijk, J. S.; Strasberg, D.; Thebaud, C. J. Biogeogr. 2010, 37, 1227-1238.

(14) Lopez, J. A.; Lin, F. T.; Duah, F. K.; Aly, Y.; Schiff, P. L. J. Nat. Prod. 1988, 51, 754-759.

(15) Tewari, S.; Bhakuni, D. S.; Dhar, M. M. Phytochemistry 1972, 11, 1149-1152.

(16) Bracher, F.; Eisenreich, W. J.; Muhlbacher, J.; Dreyer, M.; Bringmann, G. J. Org. Chem. 2004, 69, 8602-8608.

(17) Chambers, C.; Stuart, K. L. Chem. Commun. 1968, 328-329. 
(18) Vanbeek, T. A.; Verpoorte, R.; Svendsen, A. B. J. Nat. Prod. 1983, 46, 226-231.

(19) Soni, V. K.; Yadav, D. J.; Bano, N.; Dixit, P.; Pathak, M.; Maurya, R.; Sahai, M.; Jain, S. K.; Misra-Bhattacharya, S. Fitoterapia 2012, 83, 110-116.

(20) Elnaas, A. R.; Grice, D.; Han, J. Y.; Feng, Y. J.; Capua, A. D.; Mak, T.; Laureanti, J. A.; Buchko, G. W.; Myler, P. J.; Cook, G.; Quinn, R. J.; Liu, M. M. Molecules 2020, 25, 2384-2395.

(21) Darby, C. M.; Nathan, C. F. J. Antimicrob. Chemother. 2010, 65, $1424-1427$.

(22) He, W. N.; Liu, M. M.; Huang, P.; Abdel-Mageed, W. M.; Han, J. Y.; Watrous, J. D.; Nguyen, D. D.; Wang, W. Z.; Song, F. H.; Dai, H. Q.; Zhang, J. Y.; Quinn, R. J.; Grkovi, T.; Luo, H. W.; Zhang, L. X.; Liu, X. T. Synth. Syst. Biotechno. 2016, 1, 187-194. 